medRxiv preprint doi: https://doi.org/10.1101/2021.09.24.21264020; this version posted September 27, 2021. The copyright holder for this preprint (which was not certified by peer review) is the author/funder, who has granted medRxiv a license to display the preprint in perpetuity.

It is made available under a CC-BY-NC 4.0 International license .

\title{
N-acetyl-L-leucine improves symptoms and functioning in GM2 Gangliosidosis (Tay-Sachs \& Sandhoff)
}

Authors Names: Kyriakos Martakis $\mathrm{MD}^{1,2}$, Jens Claassen $\mathrm{MD}^{3}$, Jordi Gascon-Bayarri $\mathrm{MD}^{4}$, Nicolina Goldschagg MD ${ }^{5}$, Andreas Hahn MD ${ }^{1}$, Anhar Hassan MBBCh ${ }^{6}$, Anita Hennig MD ${ }^{4}$, Simon A. Jones MD ${ }^{7}$, Heather Lau MD ${ }^{8}$, Susan Perlman MD ${ }^{9}$, Reena Sharma $\mathrm{MD}^{10}$, Susanne A. Schneider $\mathrm{MD}^{5}$, Tatiana Bremova-Ertl MD ${ }^{11}$

\section{Affiliations:}

1. Department of Pediatric Neurology, University Children's Hospital (UKGM) and Medical Faculty, Justus Liebig University of Giessen, Giessen, Germany

2. Department of Pediatrics, Medical Faculty and University Hospital, University of Cologne, Cologne, Germa

3. Department of Neurology, Essen University Hospital, University of Duisburg-Essen, Germany; Department of Neurocritical Care, Neurological and Neurosurgical First Stage Rehabilitation and Weaning, MediClin Klinik Reichshof, Germany.

4. Department of Neurologic Diseases and Neurogenetics, Institut d'Investigació Biomèdica de Bellvitge, Barcelona, Spain

5. Department of Neurology, Ludwig Maximilian University of Munich, Germany

6. Department of Neurology, Mayo Clinic, Rochester, MN, United States

7. Willink Unit, Manchester Centre for Genomic Medicine, Royal Manchester Children's Hospital, University of Manchester, United Kingdom

8. Division of Neurogenetics, New York University Langone, NY, United States

9. Department of Neurology, University of California Los Angeles, CA, United States

10. Department of Adult Metabolic Medicine, Salford Royal Foundation NHS Trust, United Kingdom

11. Department of Neurology, University Hospital Bern (Inselspital), Switzerland 


\begin{abstract}
Background and Objective: GM2 gangliosidosis (Tay-Sachs and Sandhoff diseases) are rare, inherited neurodegenerative disorders with no available symptomatic or disease modifying treatments. This clinical trial aimed to investigate the safety and efficacy of N-acetyl-L-leucine (NALL) on symptoms of pediatric ( $\geq 6$ years) and adult patients with GM2 gangliosidosis.

Methods: We conducted an 8-center, multi-national, open-label, rater-blinded Phase IIb study (IB1001-201). Patients with a genetically confirmed diagnosis of GM2 gangliosidosis were assessed during a baseline period, a 6-week treatment period (orally administered NALL 4 g/day in patients $\geq 13$ years, weight-tiered doses for patients $6-12$ years), and a 6 -week post-treatment washout period. The primary Clinical Impression of Change in Severity (CI-CS) endpoint (based on a 7-point Likert scale) was assessed by blinded, centralized raters who compared randomized video pairs of each patient performing a pre-defined primary anchor test (8-Meter Walk Test or 9Hole Peg Test) during each study periods. Secondary outcomes included cerebellar rating scales (namely Scale for the Assessment and Rating of Ataxia), clinical global impression, and quality of life assessments.
\end{abstract}

Results: 30 patients aged 6 to 55 years with a confirmed diagnosis of GM2 gangliosidosis (TaySachs or Sandhoff's disease) were enrolled. 29 patients were included in the primary modified intention-to-treat analysis. NALL met the CI-CS primary endpoint (mean difference 0.71, $\mathrm{SD}=2.09,90 \%$ CI $0.00,1.50, p=0.044$ ), as well as secondary endpoints. No treatment-related serious adverse events occurred.

Conclusions: This study showed NALL led to a statistically significant improvement in symptoms, functioning, and quality of life in patients with GM2 gangliosidosis. It is a safe, welltolerated, easily administered oral therapy, therefore offering a favorable risk/benefit profile for this serious, debilitating disorder. NALL is a new therapeutic option for the treatment of this rare disease that has no other approved therapies worldwide.

Classification of Evidence: This study provides Class IV evidence NALL is safe, well-tolerated, and improves neurological symptoms and quality of life in patients with GM2 gangliosidosis.

Trial Registration Information: The trial is registered with ClinicalTrials.gov (NCT03759665; registered 30-Nov-2018), EudraCT (2018-004406-25), and DRKS (DRKS00017539). The first patient was enrolled 07-June-2019. 


\section{Introduction}

GM2 gangliosidosis, i.e. Tay-Sachs and Sandhoff diseases are rare (incidence 0.28:100,000), autosomal-recessive lysosomal storage disorders. ${ }^{1}$ The disease typically begins in early childhood, is chronic, progressive, severely reduces the quality of life, and is prematurely fatal. The clinical presentation is characterized by broad heterogeneity in systemic, psychiatric, and neurological symptoms, which depend on the age of onset of neurological symptoms. ${ }^{2}$ There are currently no approved treatments for GM2 gangliosidosis in any jurisdiction worldwide.

$\mathrm{N}$-acetyl-L-leucine (NALL) is the L- enantiomer of N-acetyl-DL-leucine, a modified amino acid that has been available in France since 1957 as a treatment for acute vertigo under the trade name Tanganil $^{\mathrm{TM}}$. Prior observational studies assessing the effect of N-acetyl-DL-leucine in patients with GM2 gangliosidosis, and other lysosomal storage disorders like Niemann-Pick disease type C (NPC) suggest a beneficial symptomatic, and long-term neuroprotective, disease-modifying treatment effect. ${ }^{3-6}$ Recently, a parallel, multinational, Phase IIb clinical trial with NALL for NPC demonstrated a statistically significant (primary and secondary endpoints) and clinically meaningful improvement in symptoms, functioning, and quality of life for children and adults with NPC. ${ }^{7}$ In all observational and clinical studies, NALL has been well tolerated with no serious side effects.

Animal studies in the GM2 mouse model $\left(\mathrm{Hexb}^{-/}\right)$and related NPC (NPC $\left.{ }^{-/-}\right)$have shown that Nacetyl-leucine significantly reduced ataxia when administered pre-symptomatically or symptomatically. In addition, treatment delayed the onset of functional decline (gait abnormalities, motor dysfunction), the decline in general health and condition, slowed disease progression, and prolonged survival. These studies have specifically identified the L-enantiomer as the active neuroprotective isomer of the racemate, indicating superior clinical effects when administered independently. ${ }^{6,8}$ In addition, pharmacokinetic studies suggest that the D-enantiomer could accumulate relative to the L-enantiomer during chronic administration of the racemate, which has the potential for long-term negative effects. ${ }^{9}$ Recently, it was reported that NALL is taken up and distributed to all tissues including the central nervous system by the monocarboxylate transporter (MCT1) and hydrolyzed to L-leucine, ${ }^{10}$ thereby functioning as a prodrug for delivery of L-leucine, a powerful intracellular metabolic signal of pathways such as mTORC1. ${ }^{11}$ Therefore, in this clinical trial, the aim was to evaluate the safety and efficacy of NALL on symptoms, functioning, and quality of life for pediatric and adult patients with GM2 gangliosidosis. 


\section{Methods}

\section{Standard Protocol Approvals, Registrations, and Patient Consents}

Approval for the study (clinicaltrials.gov identifier NCT03759639, EudraCT number 2018004331-71, and DR KS-ID: DRKS00016567) was obtained by National Regulatory Authorities in each country (German Federal Institute for Drugs and Medical Devices, Spain Agency of Medicines and Medical Devices, UK Medicines and Healthcare products Regulatory Agency, and US Food and Drug Administration), and the applicable responsible central research ethics committees / institutional review boards for each center (Ethics Committee of Ludwig Maximilian University of Munich (19-119), Bellvitge Hospital University Clinical Research Ethics Committee (AC004/19), North West - Greater Manchester South (260774), Mayo Clinic Institutional Review Board (19-000373), Office of Science and Research Institutional Review Board, New York University School of Medicine (i17-01666), University of California Los Angeles Institutional Review Board (19-000348)). Written informed consent was obtained for all study participants by the patient or, if applicable, their parent or legal representative.

\section{Study design}

The IB1001-202 clinical trial was one of three multi-national, open-label, rater-blinded trials that utilize a single master protocol to investigate NALL (Sponsor Code IB1001) for the treatment of three rare, neurodegenerative diseases (in addition to GM2 gangliosidosis, NPC [NCT03759639] and Ataxia-Telangiectasia [NCT03759678]). We used the same methodology study design as the one employed in a previous study and described in detail before. .,12 $^{2}$

The IB1001-201 clinical trial was separated into two study phases to enable the investigation of both the symptomatic ("Parent Study"), and long-term ("Extension Phase") safety and efficacy of NALL. The results of the Parent Study are reported below. The Extension Phase is ongoing.

\section{Participants}

Patients aged 6 years and older with a confirmed genetic diagnosis of GM2 gangliosidosis were eligible to participate. Patients taking prohibited medications (i.e. medications which may have confounded the safety or efficacy analysis of the trial, including Aminopyridines, N-acetyl-DLleucine, N-acetyl-L-leucine (prohibited if not provided as IMP), Riluzole, Gabapentin, Varenicline, Chlorzoxazone, Sulfasalazine, Rosuvastatin) were required to complete a minimum 42-day washout prior to screening. The complete eligibility criteria were previously published. ${ }^{12}$

\section{Procedures}

Adult and pediatric patients with a confirmed genetic diagnosis of GM2 gangliosidosis were recruited at 8 clinical research Universities and Hospitals in four countries (Germany, Spain, the United Kingdom and the United States) (Figure 1). The Parent Study consisted of three study periods: a baseline period (with or without a study run-in washout from prohibited medications), a treatment period, and a post-treatment washout period, with two patient visits per period. Duration of the treatment phase and washout phase were 42 days ( +7 days) each, per protocol. No randomization of participants /allocation of participants to different study arms occurred (i.e., to different intervention arms). However, patient videos obtained from each study period were randomized and assessed as the basis of the primary endpoint by blinded, centralized raters. The 
schedule of events is reported in Table 1. This study was ongoing during the Coronavirus Pandemic (COVID-19), which significantly impacted the schedule of events.

At the initial screening visit, patients were classified as either "naïve" or "non-naïve" depending on their use of prohibited medications within the past 6 weeks. "Non-naïve" patients were given the one-time opportunity to undergo a minimum of 42 days washout before returning for the baseline 1 screening visit.

During the treatment period, patients aged $\geq 13$ years or aged 6-12 years weighing $\geq 35 \mathrm{~kg}$ received $4 \mathrm{~g}$ /day of orally administered NALL (powder for suspension in $40 \mathrm{~mL}$ Ora-Blend ${ }^{\circledR}$ ) three times per day ( $2 \mathrm{~g}$ in the morning, $1 \mathrm{~g}$ in the afternoon, and $1 \mathrm{~g}$ in the evening). Patients aged 6-12 years weighing $<35 \mathrm{~kg}$ received weight-tiered doses two or three times per day based on approximately $0.1 \mathrm{~g} / \mathrm{kg} /$ day.

After their final visit of the Parent Study (Visit 6), patients were allowed to enter a long-term, open-label extension study which is ongoing.

\section{Outcomes}

The primary endpoint was the novel, functionally relevant Clinical Impression of Change in Severity (CI-CS). The CI-CS assessment was performed by blinded, centralized raters who compared paired videos of each patient performing a "primary anchor test" (either the 8 Meter Walk Test (8MWT) or 9 Hole Peg Test - Dominant Hand (9HPT-D)) at baseline (Visit 2), end of treatment (Visit 4), and end of washout (Visit 6) study visits. Blinded to the time point of each video in a given pair, the raters made an objective comparison scored on a 7-point Likert scale of the change in the severity of the patient's neurological signs and symptoms from Video 1 to Video 2. Details of CI-CS administration and assessment have been previously published. ${ }^{12}$

Each patient's primary anchor test (8MWT or 9HPT-D) was selected by the Principal Investigator at Visit 1 based on the patient's unique clinical symptoms to better ensure the clinical relevancy of the primary outcome assessment. The anchor tests were filmed in a standardized way at each visit and uploaded for centralized review. A pool of three board-certified neurologists performed the central video analysis. Two "primary raters" were responsible for the initial comparison of the video pairs. For cases where the two initial reviewers differed in their assessment of the primary CI-CS score by more than one (1) point, the third rater acted as an adjudicator.

The raters assessed: "Compared to the first video, how has the severity of the patient's performance on the 8MWT or 9HPT-D changed (improved or worsened) as observed in the second video?" The CI-CS assessment was based on a 7-point Likert scale ranging from +3 (significantly improved) to -3 (significantly worse).

Secondary assessments were assessed and analyzed between two time points: (i) the "treatment period", baseline (Visit 2) to the end of treatment (Visit 4); (ii) the "washout period", the end of treatment (Visit 4) to the end of post-treatment washout (Visit 6).

Given the lack of a validated disease-specific rating scale, the modified Disability Rating Scale (mDRS) was applied, to evaluate the overall neurologic status in GM2 gangliosidosis, which 
consists of 6 subdomains (ambulation, manipulation, Seizures, Language, Swallowing, Ocular movements). ${ }^{13}$ Cerebellar function evaluations were administered, including: (1) the Scale for the Assessment and Rating of Ataxia (SARA), an 8-item clinical rating scale (gait, stance, sitting, speech, fine-motor function, and coordination; range $0-40$, where 0 is the best neurologic status and 40 the worst); ${ }^{14}$ and (2) the Spinocerebellar Ataxia Functional Index (SCAFI), comprising 8$\mathrm{m}$ walking time performed by having patients walk twice, as quickly as possible, from one line to another excluding turning, the 9-Hole Peg Test (9HPT) with the dominant and non-dominant hand, and the number of "PATA" repetitions over 10 seconds (PATA). ${ }^{14}$ As reported, the quantifiable time-based SCAFI assessments do not best capture clinically meaningful changes in functioning or quality of life; hence, the CI-CS assessment was developed. ${ }^{12}$

Subjective impairment and quality of life were evaluated by using the Clinical Global Impression of Severity and Improvement Scale (completed by the Investigator, Caregiver, and Patient), ${ }^{15}$ the EuroQol (EQ) 5Q-5D-5L/Y questionnaire and the visual analog scale (VAS). ${ }^{16}$

Safety Assessments included adverse event monitoring, clinical laboratory testing and sparse pharmacokinetic sampling, and collecting vital signs and electrocardiograms.

\section{Randomization and masking}

CI-CS anchor test videos (8MWT and 9HPT-D) were submitted directly from trial sites to a thirdparty vendor, Medpace Core Laboratories (MCL). For each patient, MCL generated a random number (1-6) via $R A N D O M . O R G$, which corresponded to a video analysis order sequence. Once all applicable videos for a patient had been obtained, MCL assigned the patient's videos to this randomization sequences, generating 3 randomized video pairs for the CI-CS assessment. These 3 CI-CS assessments were released to the blinded raters for review via the secure MCL Clintrak Imaging System Portal.

Only the MCL IB1001 study team had access to the randomization sequences. To ensure that central raters were blinded to any patient identifiers, each patient video was assigned a "barcode" and "reading number" that identified the video throughout the central review process. Usage of the "barcode" and "reading number" blinded the raters to any information (i.e., Patient ID, date of birth, visit identifier) that might have introduced bias during the central review.

\section{Statistical analysis}

The primary endpoint was defined as the numerical difference of the CI-CS value for the treatment period (Visit 2 versus Visit 4) minus the CI-CS value for the washout period (Visit 4 versus Visit $6)$. This endpoint appropriately captured improvement in symptoms during the treatment period together with worsening of symptoms during the washout period. A sample size of 30 patients would provide the trial with $76 \%$ power, at a one-sided significance level of $5 \%$, to detect a mean effect of at least 0.45 in the primary endpoint (assuming a standard deviation 1.02). Pragmatic considerations given the rarity of the neurological conditions prevented the specification of a higher level for the power. The analysis of data, however, is not dependent on the value chosen for study power.

The primary analysis was performed according to the modified intention-to-treat (mITT) principle, used to estimate the treatment effect regardless of discontinuation and to provides a perspective of 
the treatment effect across the entire population. The mITT analysis set was defined as all patients who receive at least one dose of the study drug and with one video recording during the baseline period (Visit 1 or 2, or both) and treatment period (Visit 3 or Visit 4, or both). The mITT analysis utilized a last observation carried forward approach for the primary CI-CS endpoint which implies that the CI-CS value for Visit 4 to Visit 6 is assigned the value 0 (stable) if both videos from the washout period (Visit 5 and Visit 6) were not available. The analysis of the primary endpoint was based on a single sample one-sided t-test comparing the mean of the CI-CS differences with zero. The null hypothesis is that the mean is $\leq 0$, with the alternative hypothesis that this mean is $>0$ and the test will be conducted at the one-sided 5\% significance level. Non-parametric $90 \%$ confidence intervals were constructed using the Hodges-Lehmann method. ${ }^{17}$ Secondary endpoints were evaluated either statistically based on a single sample t-test or a single sample Wilcoxon Signed Rank test or descriptively, as pre-defined in the Statistical Analysis Plan (SAP). There was no formal hierarchical structure defined for the secondary endpoints and results on these endpoints should therefore be viewed as exploratory. For each of the primary and secondary endpoints, there was separate analyses within key subgroups pre-defined in the SAP. The Safety Analysis Set (SAF) consisted of all patients who received at least one dose of study drug. The safety, integrity, and feasibility of the trial were monitored by an independent data safety monitoring board (DSMB) consisting of three independent, non-participating members (including two clinicians and a statistician).

\section{Data Availability}

All authors had full access to all the data in the study and had final responsibility for the decision to submit for publication. The study Sponsor, IntraBio Ltd. is committed to providing qualified scientific researchers appropriate access to anonymized data and clinical study information from the company's clinical trials for the purpose of conducting legitimate scientific research. Requests for specific data will be considered along with the rationale, description of use need, and clinical value of the proposed analysis. IntraBio supports an approach to sharing data that responsibly reflects the interests of all parties involved in clinical trials, including protecting the rights and privacy of trial participants, the innovator's intellectual property rights, and other incentives for innovation, and as such, will evaluate requests for sharing company clinical trial data with qualified external scientific researchers. Requests to access the data from this clinical trial may be made at info@intrabio.com. Data will be made available for request after product approval in the United States and European Union, after product development is discontinued, or as otherwise required by law or regulation. There are circumstances that may prevent IntraBio from sharing the requested data as the product is investigational at this time. 


\section{Results}

\section{Study Population}

Between 07 June 2019 and 01 October 2020, thirty-six participants were screened, and thirty patients were enrolled per-protocol (Figure 1). The demographic and baseline clinical characteristics of the enrolled patients are presented in table 2. Twenty-nine patients qualified for the primary mITT analysis set $(96.7 \%)$, which included all patients dosed who had at least one treatment visit (Visit 3 or Visit 4). One patient was withdrawn after Visit 2 due to a self-reported tremor they believed was related to the IMP. The patient did not participate in a follow-up or early termination visit, and thus the Principal Investigator was unable to assess the patient in person/ further evaluate the causality of this AE. Another patient was withdrawn after Visit 3, as they were unwilling to travel due to COVID-19.

The median duration of IB1001 treatment was 49 days (range: 16-132), with a mean duration of exposure of 58.8 days. The study was ongoing during the COVID-19 pandemic, which did impact the schedule of events, including the duration of treatment /post-treatment washout period for select patients, meaning patients were dosed / on washout until it was safe and feasible to conduct an on-site end of treatment/washout visit in adherence with all COVID-19 local regulations. These deviations due to COVID-19 are reflected by the limited size of the per protocol population $(\mathrm{n}=$ 19). Twenty-seven patients $(90.0 \%)$ completed the Parent Study (Visit 6) (last patient last visit occurred 07 January 2021).

\section{Efficacy}

The CI-CS primary endpoint of the study reached statistical significance with $p=0.039$ with mean value $=0.71(\mathrm{SD}=2.09$, median=1.0) and Hodges-Lehmann 90\% confidence interval (CI) $(0.00$, 1.50). There were no missing values for CI-CS for the treatment period although there were two missing values for CI-CS during the washout period (Figure 2).

The CI-CS component for the treatment period had a mean value of $0.34(\mathrm{SD}=1.59$ median=0.5), showing on average an improvement in the patients' condition over that period, while the CI-CS component for the washout period had a mean value of $-0.36(\mathrm{SD}=1.33$, median $=-0.50)$ showing on average a worsening. There was no difference observed between the CI-CS comparing the baseline and washout visits (Visit 6 versus Visit 2): mean value $0.063(\mathrm{SD}=1.32$, median $=0$, $\mathrm{n}=30$ ). This reinforced the treatment effect, but also demonstrated the absence of a learning effect on the CI-CS anchor tests.

The inter-rater correlation of the CI-CS scores was calculated for 81 pairs of videos recorded in the trial. The Spearman Rank correlation was $83 \%$, indicating a high degree of consistency in the two blinded raters' assessment of the videos. This result was comparable with the inter-rater correlation of $70 \%$ in the NALL clinical trial for NPC (IB1001-201). ${ }^{7}$ In the IB1001-201 (NPC) and IB1001-202 (GM2 gangliosidoses) trials, different primary and adjudication raters were used. That this degree of consistency is produced is supportive of the robustness and consistency of the CI-CS methodology.

A Forest plot (Figure 3) is included to display results for the primary endpoint in the pre-defined subgroups showing Hodges-Lehman median estimates and corresponding $90 \%$ confidence 
intervals calculated where possible. There is some variation as would be expected given the small sample sizes but there is no evidence that would suggest a differential treatment benefit across the population as a whole. Instead, across the subgroups, there was a consistent trend of improvement during treatment and deterioration during the washout.

The CGI-Change assessment is presented around a reference value of $0=$ no change, so that for example $-1=$ minimally worse and $+1=$ minimally improved. The investigator, caregiver, and patient CGI-C were consistent and showed an average improvement during the treatment period and deterioration during the washout period. For the investigator's CGI-C, the mean change during the treatment period was $0.8(\mathrm{SD}=1.0$, median=1.0, $\mathrm{n}=28)$ with $90 \% \mathrm{CI}(0.5,1.0) p<0.001$ while the mean change during the washout period was $-0.9(\mathrm{SD}=0.8$, median $=-1.0, \mathrm{n}=27)$ with $90 \% \mathrm{CI}$ $(-1.0,-0.5) p<0.001$ (Figure 4A). For the caregiver's CGI-C, the mean change during the treatment period was $0.8(\mathrm{SD}=1.2$, median $=1.0, \mathrm{n}=26$ with $90 \% \mathrm{CI}(0.5,1.5) p=0.001$, while the mean change during the washout period was $-0.8(\mathrm{SD}=1.0$, median $=-1.0, \mathrm{n}=25)$ with $90 \% \mathrm{CI}(-1.0$, $0.5) p<0.001$ (Figure 4B). For the patient's CGI-C, the mean change during the treatment period was $1.0(\mathrm{SD}=1.1$, median=1.0, $\mathrm{n}=28)$ with $90 \% \mathrm{CI}(0.5,1.5) p<0.001$, while the mean change during the washout period was $-0.6(\mathrm{SD}=1.2$, median $=-1.0, \mathrm{n}=27)$ with $90 \% \mathrm{CI}(-1.0,0.0) p=0.01$ (Figure 4C).

The mean total SARA score at baseline (Visit 2) was $14.24(\mathrm{SD}=7.29$, median=12.50, $\mathrm{n}=29)$ and encompassed a full range of disease-severity $(\min =5, \max =33)$. The mean change in SARA score during the treatment period was $-1.41(\mathrm{SD}=1.67$, median $=-1.50, \mathrm{n}=28)$ with $90 \% \mathrm{CI}(-1.75,-0.75)$ $p<0.001$, demonstrating an improvement on cerebellar signs and neurological symptoms. The mean change in SARA score during the washout period was $1.43(\mathrm{SD}=2.08$, median $1.00, \mathrm{n}=27)$ with $90 \%$ CI $(0.50,2.00), p<0.001$, showing a deterioration (Figure 5A). There was no difference observed between the baseline visit to the washout visit (Visit 2 to Visit 6); the mean change in score was $0.04(\mathrm{SD}=1.88$, median=0, $\mathrm{n}=27)$ with $90 \% \mathrm{CI}(0.65,-0.58) p=0.468$. This further reinforced the treatment effect, but also demonstrated the absence of a learning effect on the SARA subdomains.

The mean mDRS at baseline (Visit 2) was $9.3(\mathrm{SD}=4.4$, median=9, $\mathrm{n}=29)$. Change from baseline through to Visit 4 showed improvement in terms of disability on average with a mean change of $0.03(\mathrm{SD}=0.75$, median=-0.21, $\mathrm{n}=28), 90 \% \mathrm{CI}(-0.063,0.00) p=0.020$ with a mean change from Visit 4 to Visit 6 (washout) of 0.042 ( $\mathrm{SD}=0.58$, median $=0.042, \mathrm{n}=27), 90 \% \mathrm{CI}(0.021,0.0635)$, $\mathrm{p}<0.001$ on average showing a worsening in terms of disability (Figure 5B).

The mean total SCAFI score at baseline (Visit 2) was $-0.318(\mathrm{SD}=0.986$, median=-0.083, $\mathrm{n}=27)$. The mean score during the treatment period was $0.016(\mathrm{SD}=0.275$, median $=0.032, \mathrm{n}=26)$ with $90 \% \mathrm{CI}(-0.033,0.104) p=0.210$. The mean change in score during the washout period was -0.008 $(\mathrm{SD}=0.242$, median $-0.009, \mathrm{n}=25)$ with $90 \%$ CI $(-0.086,0.026) p=0.315$ (Figure 6A). Of the SCAFI domains, there was a notable change in the PATA test, demonstrating a clear improvement in speech. The mean change in PATA score during the treatment period was $1.65(\mathrm{SD}=2.49$, median $=1.50 \mathrm{n}=26$ ) with $90 \%$ CI $0.75,2.50) p<0.001$. The mean change in PATA score during the washout period was $-1.12(\mathrm{SD}=2.74$, median $-1.0, \mathrm{n}=25)$ with $90 \% \mathrm{CI}-2.00,0.00) p=0.027$ (Figure 6B). 
medRxiv preprint doi: https://doi.org/10.1101/2021.09.24.21264020; this version posted September 27, 2021. The copyright holder for this preprint (which was not certified by peer review) is the author/funder, who has granted medRxiv a license to display the preprint in perpetuity.

It is made available under a CC-BY-NC 4.0 International license .

The results of EQ-5D-5L/ EQ-5D-Y and EQ-VAS were summarized by visit using descriptive statistics, consistent with the questionnaires. Of the domains, there was a trend for improvement in patient's mental health, as evaluated by the anxiety/depression domain (Figure 7).

\section{Safety}

A treatment-emergent adverse event (TEAE) was any adverse event (AE) that appeared or worsened after study treatment began (i.e. in the treatment or washout period). Eight related AEs were reported for 4 patients, including: headache, nausea followed by vomiting (once), flatulence, asthenia, acne, and tremor. The events were transient and manageable. No serious adverse reactions were reported. No deaths occurred during the study. Results of plasma and urine tests, vital signs, and ECG recordings were normal or rated as clinically non-significant. Adherence was high as shown by treatment compliance and the regular urine analyses for prohibited medications. 


\section{Discussion}

Here we report the positive results of a Phase IIb clinical trial investigating the safety and efficacy of the modified amino acid NALL in patients aged 6 years or older with GM2 gangliosidosis. The major findings of the IB1001-202 trial are: first, NALL improved cerebellar signs, fine motor function, stance and gait in patients with GM2 gangliosidosis, and this improvement in neurologic status also led to an improvement in functioning and quality of life. Second, improvement of neurological status was observed across a broad demographics of patients (i.e. age, gender, age of onset, disease severity, etc.) indicating NALL may be an applicable treatment for all patients with GM2 gangliosidosis. Third, the low frequency of AEs ( 8 related AEs in 4 patients of 30 participants) and their transient, manageable nature inform a favorable benefit-risk profile.

IB1001-202 is the second multinational, Phase IIb clinical trial to be completed with NALL. The results of the trial are consistent with a parallel trial completed for NPC, where treatment also had a statistically significant and clinically meaningful effect on the primary CI-CS and secondary (SARA, CGI-C, etc) endpoints. ${ }^{7}$

As briefly described, these clinical findings correlate directly with studies in the GM2 gangliosidosis mouse model $\left(\mathrm{Hexb}^{-/}\right)$and NPC mouse model $(\mathrm{NPC}-/)$ where $\mathrm{N}$-acetyl-leucine reduced ataxia when treatment was commenced pre-symptomatically (from 3-weeks of age onward) or symptomatically (for 1-week treatment, starting at 8-weeks of age). NALL restored aerobic (pyruvate dihydrogenase-dependent) and enhanced anaerobic (lactate dehydrogenasedependent) glycolysis, and returned the glutamate-metabolizing enzyme, glutamate dehydrogenase, to levels observed in $\mathrm{Hexb}^{+/+}$and NPC ${ }^{+/+}$null mice. ${ }^{6,8}$ In normalizing altered glucose and glutamate metabolism, NALL improves energy production, as well as cellular functions and signaling. This is manifested via the drugs modulation of multiple secondary therapeutic targets, including a reduction in lipid and cholesterol accumulation and lysosomal volume, ${ }^{6,18}$ a reduction in neuroinflammation, ${ }^{19}$ and a normalization of neuronal membrane potential. ${ }^{20}$ These multimodal actions lead to the restoration of neuronal function and improvement of overall brain health, including throughout the cerebellum. These effects correlate to NALLs symptomatic effects (e.g. improvement of postural stability, gait, fine motor function with diadochokinesia and speech) and neuroprotective action, respectively. The ongoing extension trial investigates NALL`s ability to slow, or even stabilize neurodegeneration, and the corresponding clinical neurological deterioration, respectively.

\section{Limitations}

The IB1001-202 parent study has several limitations. First, the study was not placebo-controlled. As previously reported, the widespread, unlicensed use of the commercially available racemate (N-acetyl-DL-leucine; Tanganil ${ }^{\mathrm{TM}}$ ) and even, patient's access to chemical grade substitutes of NALL, within the GM2 gangliosidosis community is documented. ${ }^{21}$ This limited the feasibility of including a placebo-control, given patients and families reluctance to participate in a study where they would be required to washout from this unlicensed medication and receive an inactive treatment for even $50 \%$ of the time. Accordingly, the study methodology aligned with principles aiming at minimizing the exposure of patients with orphan diseases - and especially pediatric patients - to placebo. ${ }^{22}$ To ensure the study minimized bias, controls were put in place for the study - including the centralized, blinded review paradigm and use of intra-patient internal control - to enable an assessment of the scientific integrity and validity of the results. The majority 
medRxiv preprint doi: https://doi.org/10.1101/2021.09.24.21264020; this version posted September 27, 2021. The copyright holder for this preprint (which was not certified by peer review) is the author/funder, who has granted medRxiv a license to display the preprint in perpetuity.

It is made available under a CC-BY-NC 4.0 International license .

of patients enrolled in the IB1001-202 clinical trial featured severe physical impairments in fine motor skills, balance, and gait, and mild to significant levels of cognitive impairment. Given the level of impairment, the potential for a placebo-effect which significantly altered neurological signs and symptoms is inherently reduced. However, to further minimize potential patient expectation or investigator bias, all aspects of the administration and video recording of the CI-CS anchor tests were standardized. Before any patient visit, site personnel were trained on these detailed protocols, including precise verbal instructions, encouragement, break times between test trials, and instructions on which trial to record. This standardization ensured the quality of videos assessed by the blinded raters, and thus the interpretability of the blinded-raters' CI-CS assessments.

Second, the novel CI-CS primary endpoint has not been previously used or yet validated. It was implemented, however, due to the methodological limitations of applying pre-existing ataxia scales in heterogeneous diseases, in which cases, the scales may be too broad and therefore not sensitive to capture small but meaningful functional changes. ${ }^{12,23}$ Accordingly, the CI-CS was developed to be a more clinically relevant endpoint capable of detecting clinically meaningful treatment effects. Validated, established scales, including the SARA, and CGI were also utilized in order supplement the primary efficacy analysis which also showed significant effects.

In conclusion, this study demonstrated NALL has a positive effect on symptoms of GM2 gangliosidosis, improved patients functioning and quality of life, and was safe and well-tolerated. The findings from IB1001-202 are consistent with previous non-clinical, observational, and clinical trials with NALL, and provide further evidence that NALL may be useful for patients with GM2 gangliosidosis, as well as similar progressive, life-threatening conditions with no approved medicinal treatments. 
medRxiv preprint doi: https://doi.org/10.1101/2021.09.24.21264020; this version posted September 27, 2021. The copyright holder for this preprint (which was not certified by peer review) is the author/funder, who has granted medRxiv a license to display the preprint in perpetuity.

It is made available under a CC-BY-NC 4.0 International license .

\section{Disclosures}

T. Bremova-Ertl, J. Claassen and N. Goldschagg received fees for the blinded rater services from IntraBio. All other authors declare no competing interests. All other authors report no disclosures relevant to the manuscript. 


\section{Figure legends}

Figure 1. Trial profile.

Figure 2. Results of the Primary Endpoint: Clinical Impression of Change in Severity (CI-CS). The CI-CS is based on a 7-point scale, ranging from -3, "significantly worse", 0 , "no change", to +3 to "significantly improved. All analysis based on the mITT population. The left-hand column (blue) illustrates CI-CS results comparing baseline to the end of the treatment period; the right-hand column (orange) illustrates the CI-CS results comparing the end of treatment period to the end of the washout period. the vertical extent of the column represents the $90 \%$ HodgesLehman (HL) Confidence Interval of the CI-CS; a solid line is used to indicate the Hodges-Lehman Median Estimator, and a cross symbol indicates the Mean response.

Figure 3. Forest plot for CI-CS scores for pre-defined subgroup analysis, based on the mITT population. The lines and dots in blue represent the change per subgroup on the CI-CS scores during the treatment period: end of treatment vs. baseline. The lines and dots in orange represent the change per subgroup on the CI-CS scores during the washout period: CI-CS scores end of washout vs. end of treatment. The dots represent the pseudo-medians or Hodges-Lehmann estimators, the horizontal lines represent the $90 \%$ confidence intervals. For some subgroups the number of patients was too small to calculate the confidence limits. In those cases the the result is presented as missing and no line representing the confidence interval is drawn. No last observation carried forward (LOCF) approach was used for this figure. Only values from patients with reported data are included.

Figure 4. Results of the Secondary Endpoint: Clinical Global Impression of Change (CGI-C). (4A) Physician's CGI-C. (4B) Caregiver CGI-C. (4C) Patient's CGI-C. For each category, the left-hand column (blue) illustrates CGI-C results comparing baseline to the end of the treatment period; the right-hand column (orange) illustrates the CGI-C results comparing the end of treatment period to the end of the washout period. the vertical extent of the column represents the $90 \%$ Hodges-Lehman (HL) Confidence Interval of the CGI-C; a solid line is used to indicate the Hodges-Lehman Median Estimator, and a cross symbol indicates the Mean response.

Figure 5. Results of the Secondary Endpoint (A) Scale for the Assessment and Rating of Ataxia (SARA). (B) Modified Disability Rating Scale (mDRS). The left-hand column (blue) illustrates results comparing baseline to the end of the treatment period; the right-hand column (orange) illustrates the results comparing the end of treatment period to the end of the washout period. The vertical extent of the column represents the $90 \%$ Hodges-Lehman (HL) Confidence Interval; a solid line is used to indicate the Hodges-Lehman Median Estimator, and a cross symbol indicates the Mean response.

Figure 6. Results of the Secondary Endpoint: Spinocerebellar Ataxia Functional Index (SCAFI). (6A) Total SCAFI scale. (6B) PATA domain. The left-hand column (blue) illustrates SCAFI/ PATA results comparing baseline to the end of the treatment period; the right-hand column (orange) illustrates the SCAFI/ PATA results comparing the end of treatment period to the end of the washout period. the vertical extent of the column represents the $90 \%$ Hodges-Lehman (HL) Confidence Interval of the SCAFI/ PATA; a solid line is used to indicate the Hodges-Lehman Median Estimator, and a cross symbol indicates the Mean response.

Figure 7. Results of the Secondary Endpoint: Euro-Quality of Life 5-Domain scale (EQ-5D-5L) anxiety and depression domain. Patients were asked to record their degree of depression or anxiety according to a 5-category scale ranging from "I am not anxious or depressed" to "I am extremely anxious or depressed". The percentage of respondents in each category is indicated by column heights for each of six visits. Visits 1 and 2 took place during Baseline; visits 3 and 4 during the Treatment period; and visits 5 and 6 during the Washout period. (Note that no patient responded "I am extremely anxious or depressed" at any visit so no column is shown for that category). 


\begin{tabular}{|c|c|c|c|c|c|c|c|}
\hline \multirow{3}{*}{$\begin{array}{l}\text { Period } \\
\text { Duration of the whole period } \\
\text { Visit number }\end{array}$} & \multicolumn{2}{|c|}{ Baseline Period } & \multirow{2}{*}{\multicolumn{2}{|c|}{$\begin{array}{c}\text { Treatment Period } \\
6 \text { Weeks }\end{array}$}} & \multirow{2}{*}{\multicolumn{2}{|c|}{$\begin{array}{c}\text { Wash-Out Period } \\
\text { 6 Weeks }\end{array}$}} & \multirow{2}{*}{\begin{tabular}{c|c}
15 & Early Term. \\
& 1 Day \\
\end{tabular}} \\
\hline & 1 Day & 2 Weeks & & & & & \\
\hline & Visit 1 & Visit 2 & Visit 3 & Visit 4 & Visit 5 & Visit 6 / EOS & ET \\
\hline Name of the Visit & "Screening/Bsl 1 & Baseline 2 & Treatment 1 & Treatment 2 & Washout 1 & Washout 2 & $\overline{\text { ET }}$ \\
\hline Timeline (Days) & Day-14 & Day 1, Start IMP & Day 28 & Day 42 & Day 70 & Day 84 & $X X$ \\
\hline Visit Window allowed & $n a$ & +7 days & +7 days & +7 days & +7 days & +7 days & $n a$ \\
\hline
\end{tabular}

\section{Table 1: Schedule of Assessments}

\begin{tabular}{|c|c|c|c|c|c|c|c|}
\hline Patient information and informed consent process & $\mathrm{X}$ & & & & & & \\
\hline $\begin{array}{l}\text { Inclusion / exclusion criteria, medical history, } \\
\text { patient demographics }\end{array}$ & $\mathrm{X}$ & $\mathrm{X}$ & & & & & \\
\hline Classify patient as "Naïve" or "Non-naïve" & $\mathrm{X}$ & & & & & & \\
\hline $\begin{array}{l}\text { Documentation of therapy \& concomitant } \\
\text { medications }\end{array}$ & $\mathrm{X}$ & $\mathrm{X}$ & $\mathrm{X}$ & $\mathrm{X}$ & $\mathrm{X}$ & $\mathrm{X}$ & $\mathrm{X}$ \\
\hline Vital signs & $\mathrm{X}$ & $\mathrm{X}$ & $\mathrm{X}$ & $\mathrm{X}$ & $\mathrm{X}$ & $\mathrm{X}$ & $\mathrm{X}$ \\
\hline 12-lead electrocardiogram (ECG) & $\mathrm{X}$ & & $\mathrm{X}$ & & $\mathrm{X}$ & & $\mathrm{X}$ \\
\hline Urine test for N-Acetyl-D-Leucine & $\mathrm{X}$ & $\mathrm{X}$ & & & $\mathrm{X}$ & $\mathrm{X}$ & $\mathrm{X}$ \\
\hline Blood safety laboratory tests \& urinalysis & $\mathrm{X}$ & $\mathrm{X}$ & $\mathrm{X}$ & $\mathrm{X}$ & $\mathrm{X}$ & $\mathrm{X}$ & $\mathrm{X}$ \\
\hline Blood sample for sparse PK & $\mathrm{X}$ & $\mathrm{X}$ & $\mathrm{X}$ & $\mathrm{X}$ & $\mathrm{X}$ & $\mathrm{X}$ & $\mathrm{X}$ \\
\hline Quality of Life EQ-5D & $\mathrm{X}$ & $\mathrm{X}$ & $\mathrm{X}$ & $\mathrm{X}$ & $\mathrm{X}$ & $\mathrm{X}$ & $\mathrm{X}$ \\
\hline Scale for Ataxia Rating (SARA) & $\mathrm{X}$ & $\mathrm{X}$ & $\mathrm{X}$ & $\mathrm{X}$ & $\mathrm{X}$ & $\mathrm{X}$ & $\mathrm{X}$ \\
\hline Modified Disabling Rating Score (mDRS) & $\mathrm{X}$ & $\mathrm{X}$ & $\mathrm{X}$ & $\mathrm{X}$ & $\mathrm{X}$ & $\mathrm{X}$ & $\mathrm{X}$ \\
\hline $\begin{array}{l}\text { Scale for Spinocerebellar Ataxia Functional } \\
\text { Index (SCAFI) }\end{array}$ & $\mathrm{X}$ & $\mathrm{X}$ & $\mathrm{X}$ & $\mathrm{X}$ & $\mathrm{X}$ & $\mathrm{X}$ & $\mathrm{X}$ \\
\hline CI-CS Anchor Test Video Record & $\mathrm{X}$ & $\mathrm{X}$ & $\mathrm{X}$ & $\mathrm{X}$ & $\mathrm{X}$ & $\mathrm{X}$ & $\mathrm{X}$ \\
\hline Clinical Global Impression of Severity (CGI-S) & $\mathrm{X}$ & $\mathrm{X}$ & $\mathrm{X}$ & $\mathrm{X}$ & $\mathrm{X}$ & $\mathrm{X}$ & $\mathrm{X}$ \\
\hline $\begin{array}{l}\text { Clinical Global Impression of Change } \\
\text { (CGI-C) by Physician, Caregiver, Patient }\end{array}$ & & & & $\mathrm{X}$ & & $\mathrm{X}$ & $\mathrm{X}$ \\
\hline Documentation of AEs & $\mathrm{X}$ & $\mathrm{X}$ & $\mathrm{X}$ & $\mathrm{X}$ & $\mathrm{X}$ & $\mathrm{X}$ & $\mathrm{X}$ \\
\hline
\end{tabular}




\begin{tabular}{|c|c|c|}
\hline \multirow[t]{3}{*}{ Age (years) } & Mean (SD) & $27.0(15.2)$ \\
\hline & Median & 28.5 \\
\hline & Range & $6.0-55.0$ \\
\hline \multirow[t]{2}{*}{ Ethnicity, n (\%) } & Asian & $1(3.3 \%)$ \\
\hline & White & $29(96.7 \%)$ \\
\hline \multirow[t]{2}{*}{ Gender, n (\%) } & Male & $11(36.7 \%)$ \\
\hline & Female & $19(63.3 \%)$ \\
\hline \multirow[t]{2}{*}{ Age Group, n (\%) } & Paediatric (<18 years) & $10(33.3 \%)$ \\
\hline & Adult $(>=18$ years $)$ & $20(66.7 \%)$ \\
\hline \multirow[t]{4}{*}{ Dose, n (\%) } & Age $6-12$ years -15 to $<25 \mathrm{~kg}-2 \mathrm{~g}$ per day & $3(10.0 \%)$ \\
\hline & Age $6-12$ years -25 to $<35 \mathrm{~kg}-3 \mathrm{~g}$ per day & $4(13.3 \%)$ \\
\hline & Age $6-12$ years $->=35 \mathrm{~kg}-4 \mathrm{~g}$ per day & $1(3.3 \%)$ \\
\hline & Age $>=13$ years $-4 g$ per day & $22(73.3 \%)$ \\
\hline \multirow[t]{2}{*}{ Geographic Location, n (\%) } & USA & $10(33.3 \%)$ \\
\hline & Europe & $20(66.7 \%)$ \\
\hline \multirow[t]{2}{*}{ Disease, $n(\%)$} & Tay-Sachs & $27(90.0 \%)$ \\
\hline & Sandhoff & $3(10.0 \%)$ \\
\hline \multirow[t]{2}{*}{ Selected Primary Anchor Test, n (\%) } & 8 Meter Walk Test (8MWT) & $12(40.0 \%)$ \\
\hline & 9 Hole Peg Test - Dominant Hand (9HPT-D) & $18(60.0 \%)$ \\
\hline
\end{tabular}

Table 2 - Demographics and baseline characteristics of the Safety Analysis Set Populatio 


\section{Figure 1}

36 patients screened

for eligibility

2 excluded as they did not meet the inclusion criteria

4 withdrew consent prior to dosing with NALL

30 enrolled and dosed with NALL

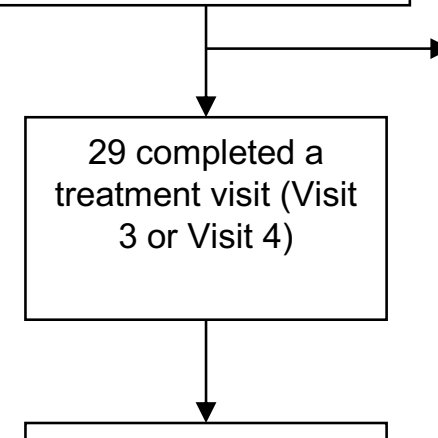

29 included in modified intentionto-treat analysis

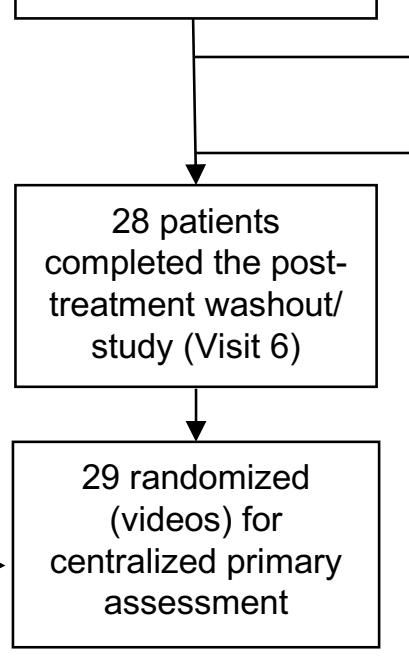

1 withdrawn after treatment (between Visit 3 and Visit 4) because they did not want to travel due to COVID-19

1 withdrawn after treatment (between Visit 5 and Visit 6 ) because they did not want to travel due to COVID-19 
Figure 2

Clinical Impression of Change in Severity

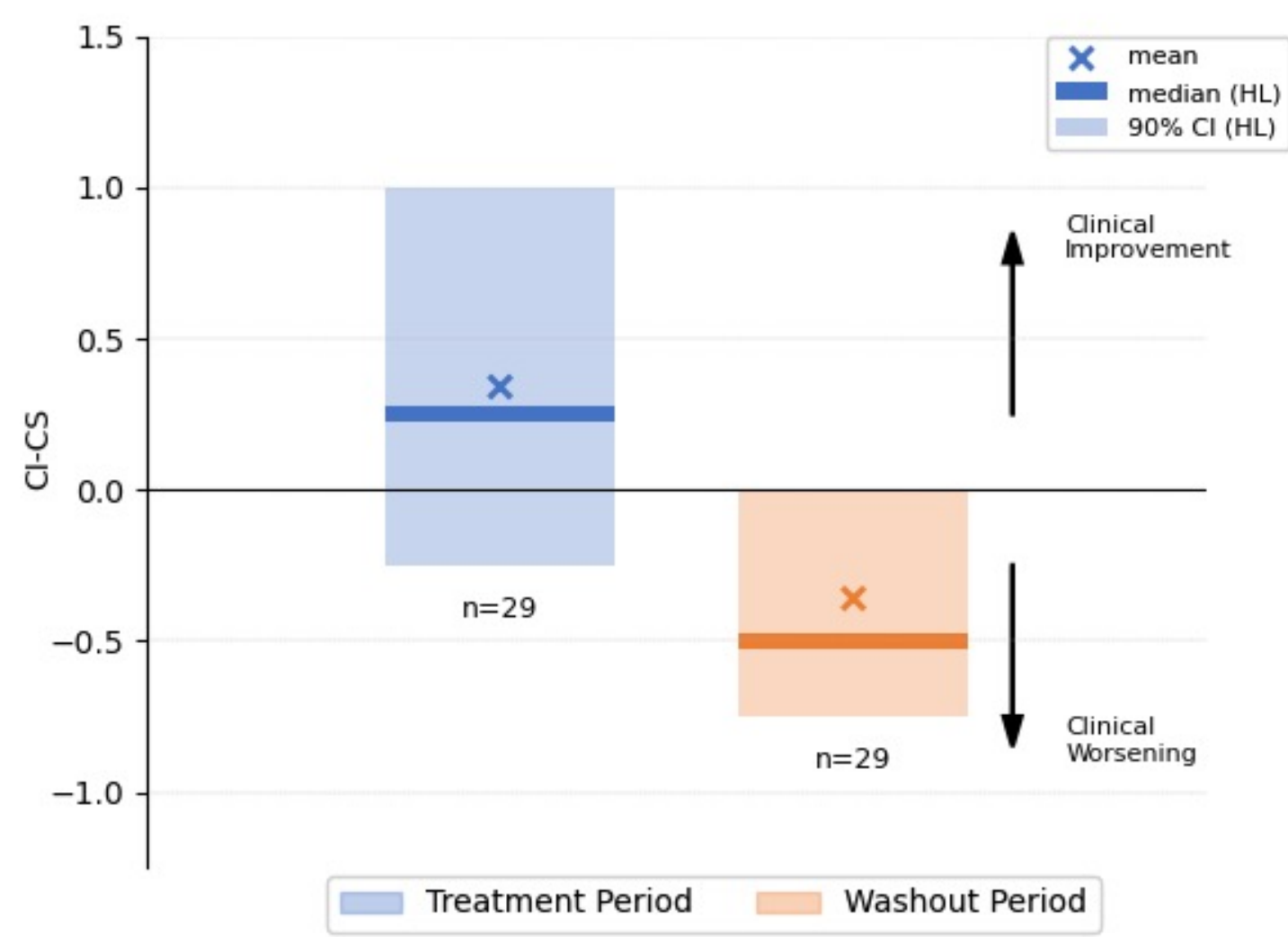




\section{Figure 3}

Subgroup

Naive yersus non-naive Non-naive

Age group

Paediatric $(<18$ years $)$

Gender

Female

Dose group

Age 6-1 2 years -15 to $<25 \mathrm{~kg}-2 \mathrm{~g}$ per day Age 6 - 12 years -25 to $<35 \mathrm{~kg}-3 \mathrm{~g}$ per day Age $6-12$ years $->35 \mathrm{~kg}-4 \mathrm{~g}$ per day

Region

Selected primary anchor test 8MWT
9HPT-D

Tay-Sachs versus Sandhoff patients Tay-Sachs

SARA Subtest Gait at Visit 1

$$
<=\text { Median (3.0) }
$$

Composite of SARA Subtests 1-4 at Visit 1 $<=$ Median $(7.0)$

ARA score at Visit 1 vs Visit 2

$>$ Median $(0.00)$

CI-S score at Visit 1 vs Visit 2

Median $(0.00)$

Age at diagnosis grou

Juvenile (2 to $<15$ years) dolescent/Late onset $(>=15$ years)

Disease severity based on SARA to tal score $>$ Median $(11.50)$
$<=$ Median $(11.50)$
N (\%)

$24(88.9 \%)$

$3(11.1 \%)$

$10(37.0 \%)$

$17(63.0 \%)$

$10(37.0 \%)$
$17(63.0 \%)$

3 (11.1\%)

$4(3.7 \%)$

$19(70.4 \%)$

$8(29.6 \%)$
$19(70.4 \%)$

$11(40.7 \%)$

$16(59.3 \%)$

$24(88.9 \%)$
$3(11.1 \%)$

$8(29.6 \%)$

$19(70.4 \%)$

$12(44.4 \%)$
$15(55.6 \%)$

$5(18.5 \%)$

$22(81.5 \%)$

$9(33.3 \%)$

$18(66.7 \%)$

$15(55.6 \%)$
$12(44.4 \%)$

$12(44.4 \%)$

$15(55.6 \%)$
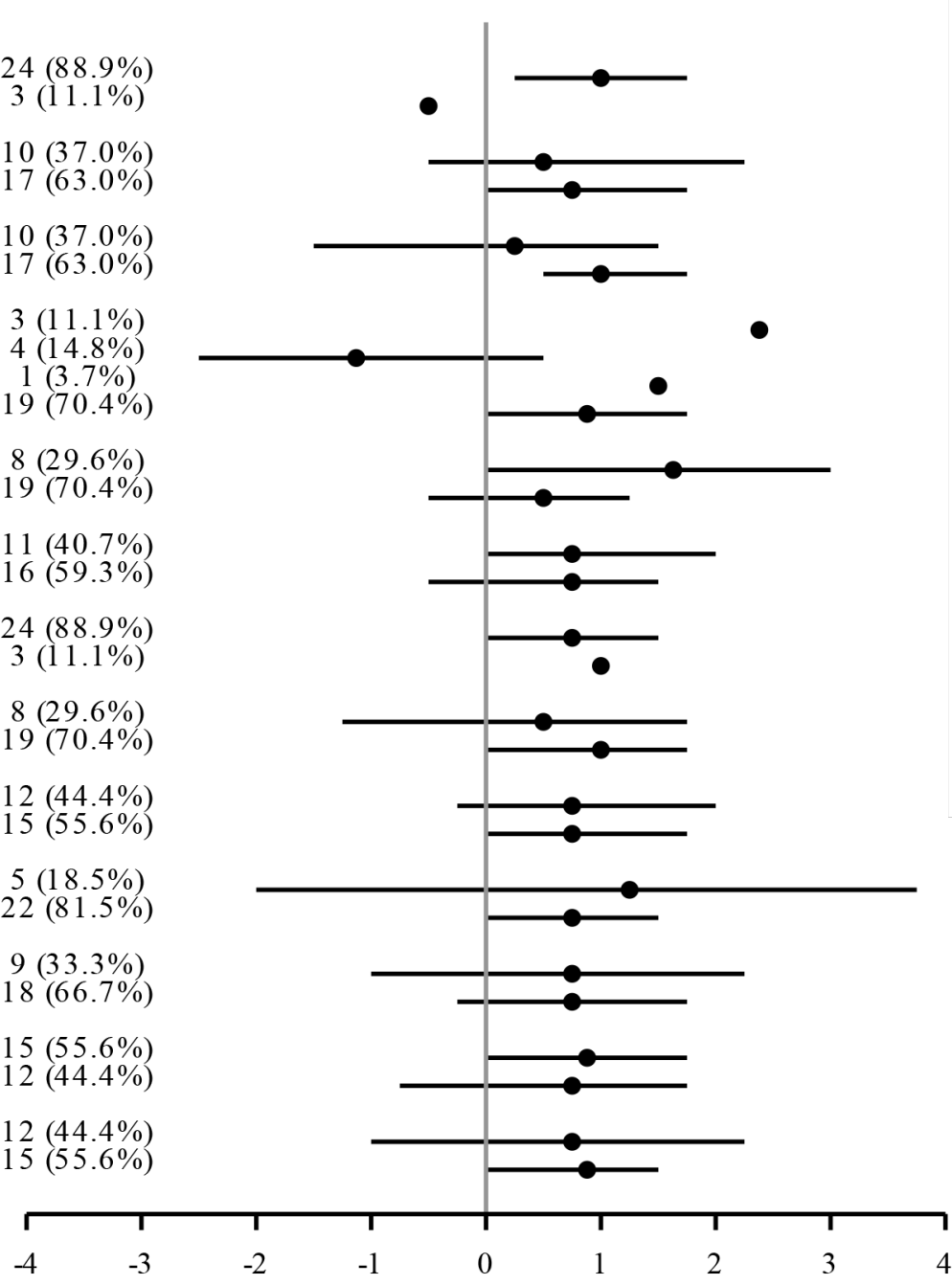

Clinical Impression of Change in Severity Difference

$$
\longleftrightarrow \quad \longrightarrow
$$

Clinical Worsening Clinical Improvement 
Figure 4A, B, C

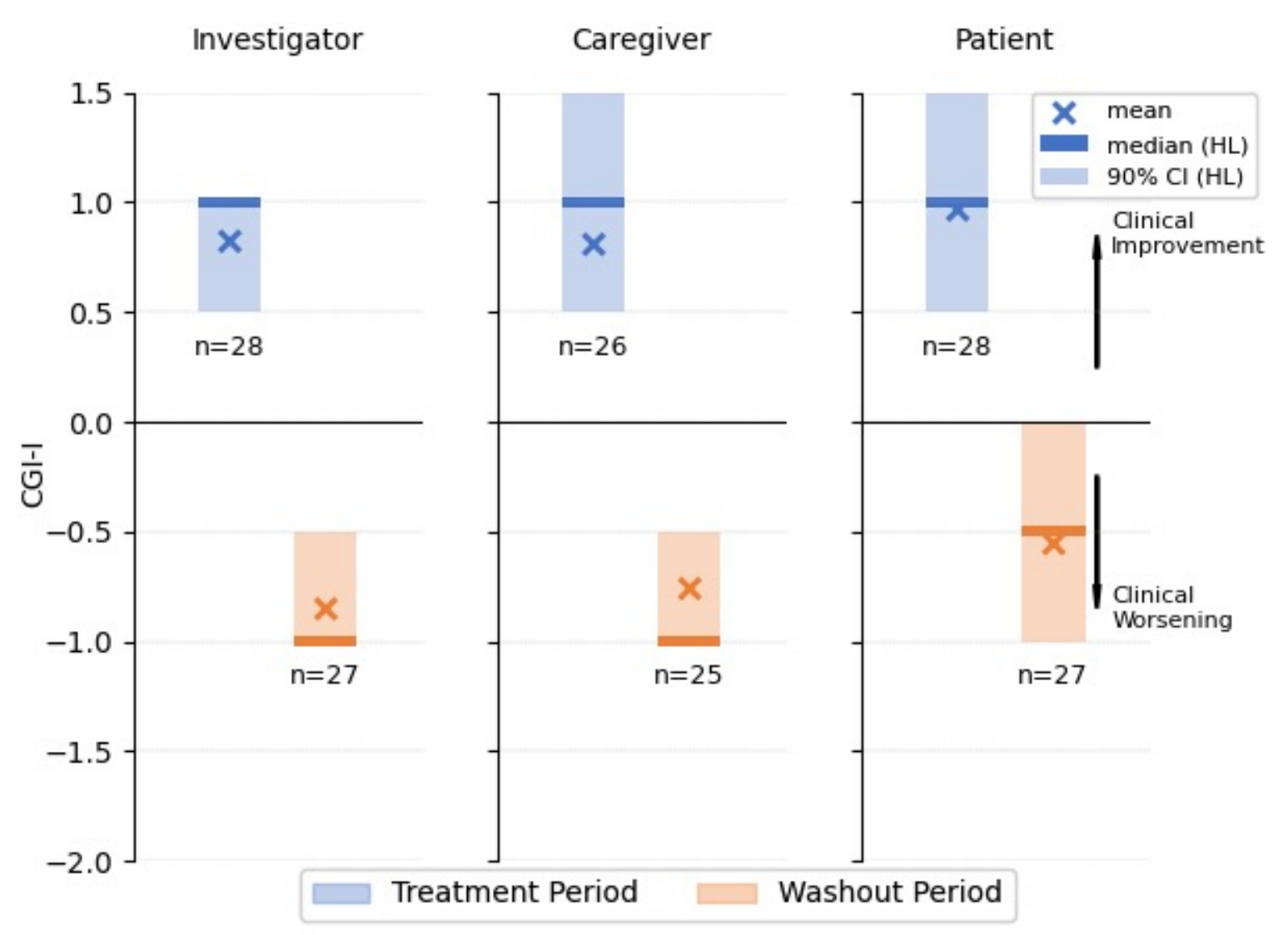




\section{Figure 5A, B}
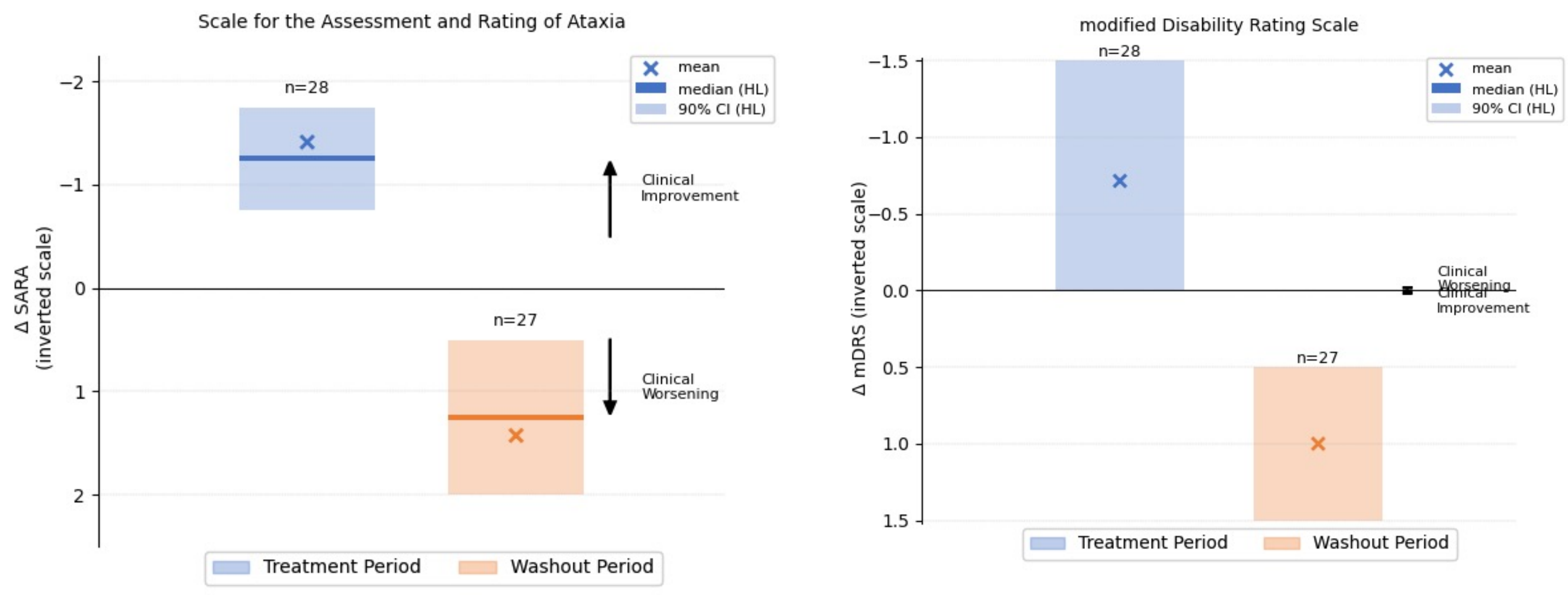
Figure 6A, B

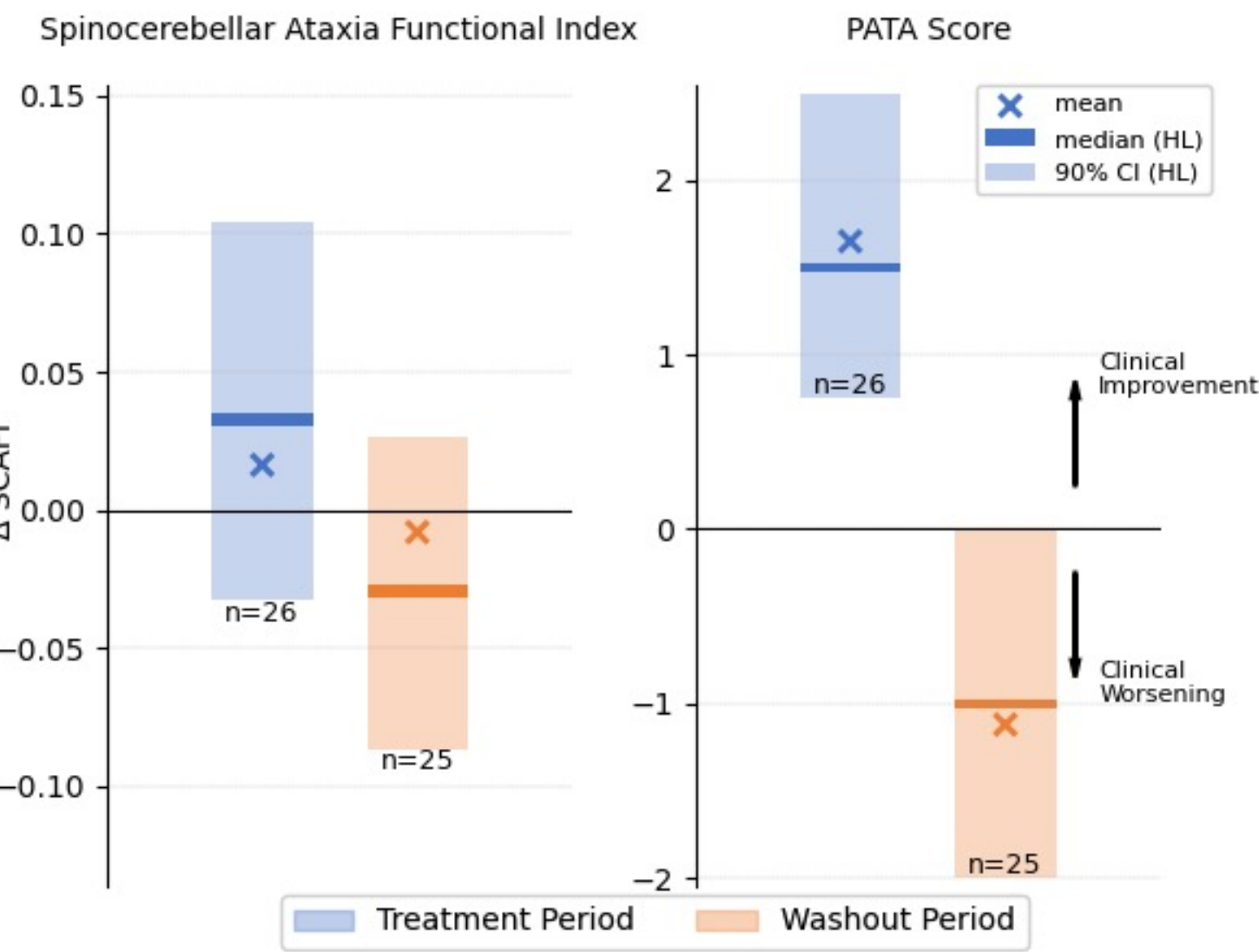


Figure 7

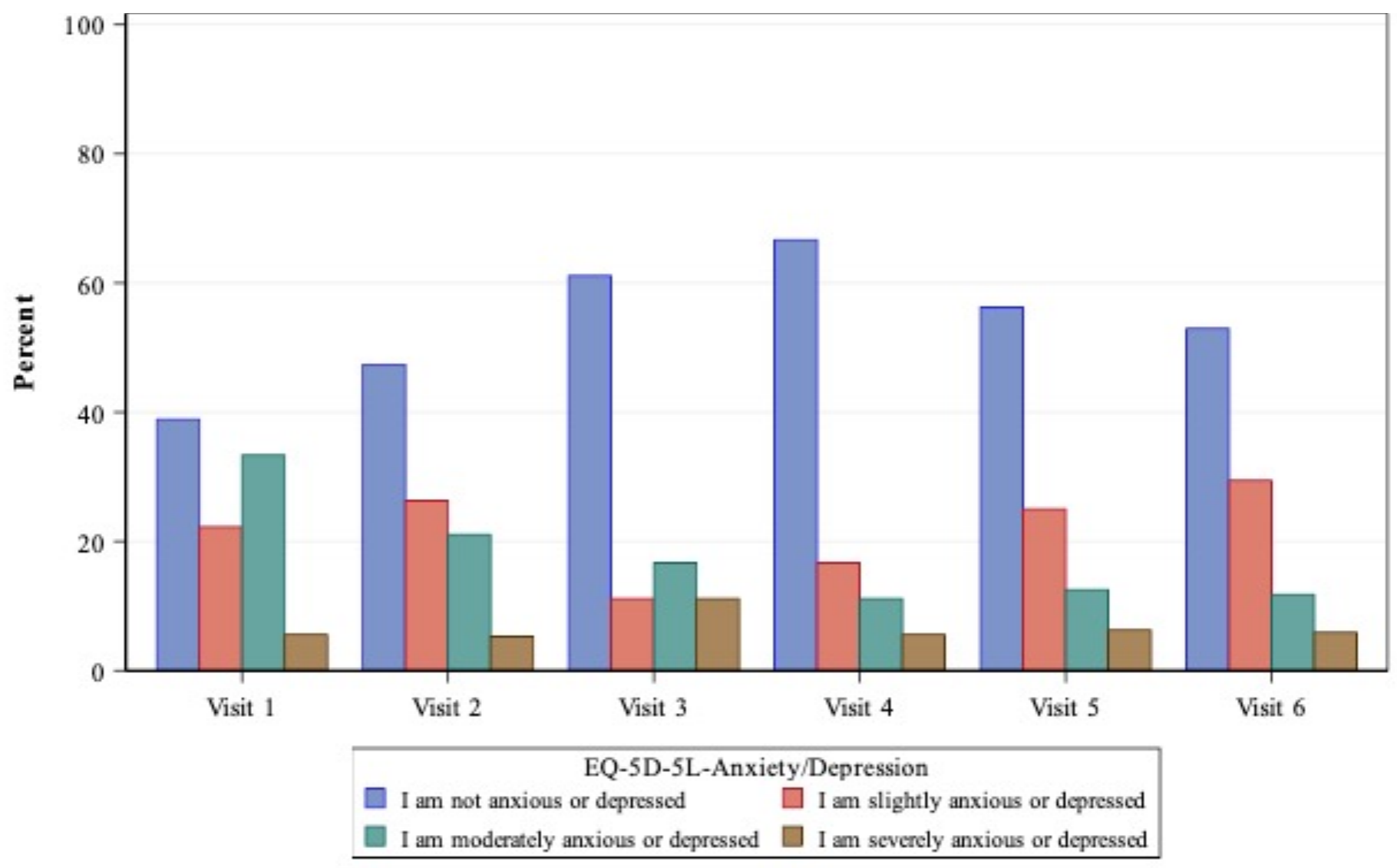




\section{References}

1. Orphanet. Prevalence and incidence of rare diseases. Epub 2017. Accessed at: https://www.orpha.net/ orphacom/cahiers/docs/GB/Prevalence_of_rare_diseases_by_alphabetical_list.pdf. Accessed February 15, 2020.

2. Cachon-Gonzalez MB, Zaccariotto E, Cox TM. Genetics and Therapies for GM2 Gangliosidosis. Curr Gene Ther. 2018;18:68-89.

3. Bremova T, Malinová V, Amraoui Y, et al. Acetyl-dl-leucine in Niemann-Pick type C: A case series. Neurology. 2015;85:1368-1375.

4. Bremova-Ertl T, Platt F, Strupp M. Sandhoff Disease: Improvement of Gait by Acetyl-DLLeucine: A Case Report. Neuropediatrics. 2020;51:450-452.

5. Cortina-Borja M, Te Vruchte D, Mengel E, et al. Annual severity increment score as a tool for stratifying patients with Niemann-Pick disease type $\mathrm{C}$ and for recruitment to clinical trials. Orphanet J Rare Dis. 2018;13:143.

6. Kaya E, Smith DA, Smith C, et al. Acetyl-leucine slows disease progression in lysosomal storage disorders. Brain Commun. 2021;3:fcaa148.

7. Bremova-Ertl T, Claassen J, Foltan T, et al. Efficacy and safety of N-acetyl-1-leucine in Niemann-Pick disease type C. J Neurol [online serial]. Epub 2021 Aug 13. Accessed at: https://doi.org/10.1007/s00415-021-10717-0. Accessed September 17, 2021.

8. Kaya E, Smith DA, Smith C, Boland B, Strupp M, Platt FM. Beneficial Effects of Acetyl-DLLeucine (ADLL) in a Mouse Model of Sandhoff Disease. J Clin Med. 2020;9:E1050.

9. Churchill GC, Strupp M, Galione A, Platt FM. Unexpected differences in the pharmacokinetics of $\mathrm{N}$-acetyl-DL-leucine enantiomers after oral dosing and their clinical relevance. PLoS One. 2020;15:e229585.

10. Churchill GC, Strupp M, Factor C, et al. Acetylation turns leucine into a drug by membrane transporter switching. Sci Rep. 2021;11:15812.

11. Ananieva EA, Powell JD, Hutson SM. Leucine Metabolism in T Cell Activation: mTOR Signaling and Beyond. Adv Nutr. 2016;7:798S-805S.

12. Fields T, Patterson M, Bremova-Ertl T, et al. A master protocol to investigate a novel therapy acetyl-L-leucine for three ultra-rare neurodegenerative diseases: Niemann-Pick type C, the GM2 gangliosidoses, and ataxia telangiectasia. Trials. 2021;22:84.

13. Iturriaga $\mathrm{C}$, Pineda $\mathrm{M}$, Fernández-Valero EM, Vanier MT, Coll MJ. Niemann-Pick C disease in Spain: clinical spectrum and development of a disability scale. J Neurol Sci. 2006;249:1-6. 
14. Schmitz-Hübsch T, du Montcel ST, Baliko L, et al. Scale for the assessment and rating of ataxia: development of a new clinical scale. Neurology. 2006;66:1717-1720.

15. Quinn TJ, Dawson J, Walters MR, Lees KR. Variability in modified Rankin scoring across a large cohort of international observers. Stroke. 2008;39:2975-2979.

16. EuroQol Group. EQ-5D Instruments | About EQ-5D. 2017. [online]. 2017. Accessed at: https://euroqol.org/eq-5d-instruments/eq-5d-51-about. Accessed February 15, 2020.

17. Hodges JL, Lehmann EL. Estimates of Location Based on Rank Tests. The Annals of Mathematical Statistics [online serial]. Institute of Mathematical Statistics; 1963;34:598611. Accessed at: https://www.jstor.org/stable/2238406. Accessed December 21, 2020.

18. te Vruchte D te, Galione A, Strupp M, Mann M. Effects of N-Acetyl-Leucine and its enantiomers in Niemann-Pick disease type C cells [online]. 2019 Oct p. 826222. Accessed at: https://www.biorxiv.org/content/10.1101/826222v1. Accessed September 17, 2021.

19. Hegdekar N, Lipinski MM, Sarkar C. N-Acetyl-L-leucine improves functional recovery and attenuates cortical cell death and neuroinflammation after traumatic brain injury in mice. Sci Rep. 2021;11:9249.

20. Vibert N, Vidal PP. In vitro effects of acetyl-DL-leucine (tanganil) on central vestibular neurons and vestibulo-ocular networks of the guinea-pig. Eur J Neurosci. 2001;13:735-748.

21. Inoue S. GM1 \& GM2 Tanganil Health Initiative Update [online]. TREND Community 2018. Accessed at: https://rend.community/news/tanganil-health-initiative-update. Accessed September 17, 2021.

22. Mulberg AE, Bucci-Rechtweg C, Giuliano J, et al. Regulatory strategies for rare diseases under current global regulatory statutes: a discussion with stakeholders. Orphanet J Rare Dis. 2019;14:36.

23. Perez-Lloret S, van de Warrenburg B, Rossi M, et al. Assessment of Ataxia Rating Scales and Cerebellar Functional Tests: Critique and Recommendations. Mov Disord. 2021;36:283-297. 